\title{
COUNTING PROCESSES WITH BERNŠTEIN INTERTIMES AND RANDOM JUMPS
}

\author{
ENZO ORSINGHER *** AND \\ BRUNO TOALDO, ${ }^{*}$ Sapienza Università di Roma
}

\begin{abstract}
In this paper we consider point processes $N^{f}(t), t>0$, with independent increments and integer-valued jumps whose distribution is expressed in terms of Bernštein functions $f$ with Lévy measure $v$. We obtain the general expression of the probability generating functions $G^{f}$ of $N^{f}$, the equations governing the state probabilities $p_{k}^{f}$ of $N^{f}$, and their corresponding explicit forms. We also give the distribution of the first-passage times $T_{k}^{f}$ of $N^{f}$, and the related governing equation. We study in detail the cases of the fractional Poisson process, the relativistic Poisson process, and the gamma-Poisson process whose state probabilities have the form of a negative binomial. The distribution of the times $\tau_{j}^{l_{j}}$ of jumps with height $l_{j}\left(\sum_{j=1}^{r} l_{j}=k\right)$ under the condition $N(t)=k$ for all these special processes is investigated in detail.
\end{abstract}

Keywords: Lévy measure; Bernštein function; subordinator; negative binomial; beta random variable

2010 Mathematics Subject Classification: Primary 60G55

Secondary $60 \mathrm{G} 50$

\section{Introduction}

In this paper we consider a class of point processes with stationary independent integervalued increments of arbitrary range. These processes can be regarded as generalizations of the Poisson process where jumps can take any positive value. Furthermore, we shall show that these processes $N^{f}(t), t>0$, can also be viewed as time-changed Poisson processes $N\left(H^{f}(t)\right)$, where $H^{f}(t)$ are subordinators associated with the Bernštein function $f$ and independent from the homogeneous Poisson process $N$ with rate $\lambda>0$. The probabilistic behavior of the processes $\mathcal{P}^{f}(t)$, with related counting processes $N^{f}(t)$, is described by the following properties:

(i) $\mathcal{P}^{f}(t)$ has independent and stationary increments;

(ii) the probability of jumps in an infinitesimal interval is given by

$$
\mathbb{P}\left\{N^{f}[t, t+\mathrm{d} t)=k\right\}= \begin{cases}\mathrm{d} t \frac{\lambda^{k}}{k !} \int_{0}^{\infty} \mathrm{e}^{-\lambda s} s^{k} v(\mathrm{~d} s)+o(\mathrm{~d} t), & k \geq 1 \\ 1-\mathrm{d} t \int_{0}^{\infty}\left(1-\mathrm{e}^{-\lambda s}\right) v(\mathrm{~d} s)+o(\mathrm{~d} t), & k=0\end{cases}
$$

Received 10 June 2014; revision received 29 October 2014.

* Postal address: Dipartimento di Statistica, Sapienza Università di Roma, Piazzale Aldo Moro 5, 00185 Roma, Italy.

** Email address: enzo.orsingher@uniroma1.it 
where

$$
f(\lambda)=\int_{0}^{\infty}\left(1-\mathrm{e}^{-\lambda s}\right) v(\mathrm{~d} s)
$$

is the integral representation of the Bernštein functions.

The Bernštein functions are $C^{\infty}$, nonnegative, and such that $(-1)^{k}\left(\mathrm{~d}^{k} / \mathrm{d} x^{k}\right) f(x) \leq 0, k \geq 1$ (see, for example, Schilling et al. (2010)). By $v$ we denote a nonnegative Lévy measure on the positive half-line such that

$$
\int_{0}^{\infty}(s \wedge 1) v(\mathrm{~d} s)<\infty
$$

We often speak of $N^{f}(t), t>0$, as a generalized Poisson process performing integer-valued jumps of arbitrary height.

These processes can be used to model many different concrete and real phenomena. For example, if we consider car accidents in the time interval $[0, t)$, the number of injured people in each crash can take any positive number. The number of clients appearing at any commercial center and arriving on different transport vehicles can also be modeled by a suitable counting process $N^{f}(t), t>0$. Analogously, in floods or earthquakes, the number of destroyed buildings in each event can be clearly of arbitrary magnitude and thus can be represented by $N^{f}(t), t>0$, with suitably chosen Bernštein function $f$ and Lévy measure $v$.

The subordinators $H^{f}$ have Laplace transforms

$$
\mathbb{E} \exp \left(-\mu H^{f}(t)\right)=\exp (-t f(\mu))=\exp \left(-t \int_{0}^{\infty}\left(1-\mathrm{e}^{-s \mu}\right) \nu(\mathrm{d} s)\right)
$$

We observe that for

$$
v(\mathrm{~d} s)=\frac{\alpha s^{-\alpha-1}}{\Gamma(1-\alpha)} \mathrm{d} s, \quad \alpha \in(0,1),
$$

we obtain the space-fractional Poisson process studied in Orsingher and Polito (2012b), where $f(\mu)=\mu^{\alpha}, \alpha \in(0,1)$. In this case, the subordinator corresponding to the space-fractional Poisson is a positively skewed stable process of order $\alpha$. If the Lévy measure is the Dirac point mass at 1 , then the corresponding subordinated Poisson process is

$$
N_{1}\left(N_{2}(t)\right), \quad t>0,
$$

where $N_{i}, i=1,2$, are independent homogeneous Poisson processes with rates $\lambda_{i}>0$. Such a process was investigated in Orsingher and Polito (2012a) and also recently in Di Crescenzo et al. (2015).

The state probabilities $p_{k}^{f}(t)=\mathbb{P}\left\{N^{f}(t)=k\right\}$ are governed by difference-differential equations of the form

$$
\frac{\mathrm{d}}{\mathrm{d} t} p_{k}^{f}(t)=-f(\lambda) p_{k}^{f}(t)+\sum_{m=1}^{k} \frac{\lambda^{m}}{m !} p_{k-m}^{f}(t) \int_{0}^{\infty} \mathrm{e}^{-s \lambda} s^{m} v(\mathrm{~d} s), \quad k \geq 0, t>0,
$$

with the usual initial conditions. From (1.4), we extract the probability generating function (PGF) $G^{f}(u, t)$ of $N^{f}(t)$ as

$$
G^{f}(u, t)=\exp (-t f(\lambda(1-u)))=\exp \left(-t \int_{0}^{\infty}\left(1-\mathrm{e}^{-s \lambda(1-u)}\right)\right) v(\mathrm{~d} s) .
$$


We prove also that

$$
\mathbb{E} u^{N\left(H^{f}(t)\right)}=\mathrm{e}^{-t f(\lambda(1-u))}
$$

and, thus, we show that

$$
N^{f}(t) \stackrel{\mathrm{D}}{=} N\left(H^{f}(t)\right) .
$$

By means of the shift operator $B^{m} p_{k}^{f}(t)=p_{k-m}^{f}(t), 0 \leq m \leq k$, we can write (1.4) as

$$
\frac{\mathrm{d}}{\mathrm{d} t} p_{k}^{f}(t)=-f(\lambda(I-B)) p_{k}^{f}(t), \quad t>0, k \geq 0,
$$

which for $f(x)=x^{\alpha}$ coincides with Orsingher and Polito (2012b, Equation 2.4). We also present a further representation of the generalized Poisson process $\mathcal{P}^{f}(t), t>0$, as the scale limit of a continuous-time random walk with steps $X_{j}$ having distribution

$$
\mathbb{P}\left\{X_{j}=k\right\}=\frac{1}{u(n)} \int_{0}^{\infty} \mathbb{P}\{N(s)=k\} v(\mathrm{~d} s), \quad k \geq n \in \mathbb{N},
$$

where $u(n)=\int_{0}^{\infty} \mathbb{P}\{N(s) \geq n\} v(\mathrm{~d} s)$. For example, for the space-fractional Poisson process the distribution (1.6) becomes

$$
\mathbb{P}\left\{X_{j}=k\right\}=\frac{\Gamma(k-\alpha) / k !}{\sum_{j=n}^{\infty} \Gamma(j-\alpha) / j !}, \quad k \geq n .
$$

We also consider the hitting-times

$$
T_{k}^{f}=\inf \left\{t \geq 0: N^{f}(t) \geq k\right\}
$$

and we show that

$$
\frac{\mathbb{P}\left\{T_{k}^{f} \in \mathrm{d} s\right\}}{\mathrm{d} s}=-\frac{\mathrm{d}}{\mathrm{d} s} \sum_{l=0}^{k-1} \frac{(-\lambda)^{l}}{l !} \frac{\mathrm{d}^{l}}{\mathrm{~d} \lambda^{l}} \mathrm{e}^{-s f(\lambda)} .
$$

We note that for $f(\lambda)=\lambda$ (the case of the homogeneous Poisson process), (1.7) yields the Erlang distribution

$$
\mathbb{P}\left\{T_{k} \in \mathrm{d} s\right\}=\lambda^{k} \mathrm{e}^{-\lambda s} \frac{s^{k-1}}{(k-1) !} \mathrm{d} s, \quad k \geq 1, s>0 .
$$

The last part of the paper is devoted to three special cases, that is,

$$
f(\mu)=\left\{\begin{array}{l}
\mu^{\alpha}, \alpha \in(0,1) \quad \text { (space-fractional Poisson process), } \\
(\mu+\theta)^{\alpha}-\theta^{\alpha}, \alpha \in(0,1) \quad \text { (tempered Poisson process), } \\
\log (1+\mu) \quad \text { (negative binomial process) }
\end{array}\right.
$$

We obtain explicitly the probability distribution $p_{k}^{f}(t), k \geq 0$, in the three cases above. Furthermore, we are able to obtain the conditional distributions, for $0<t_{1}<\cdots<t_{r}<t$,

$$
\mathbb{P}\left\{\bigcap_{j=1}^{r}\left\{\tau_{j}^{l_{j}} \in \mathrm{d} t_{j}\right\} \mid N^{f}(t)=k\right\}
$$


(where $\tau_{j}^{l_{j}}$ are the instants of occurrence of the $j$ th Poisson event with size $l_{j}$ ) for $f(\mu)=$ $\mu^{\alpha}, f(\mu)=\log (1+\mu)$. The tempered Poisson process has finite moments (unlike the space-fractional Poisson process) as well as the negative binomial of which many particular distributions can be explicitly evaluated.

The Poisson process and the negative binomial processes have been generalized in many directions; see, for example, Beghin (2013), Brix (1999), Cahoy and Polito (2012), Di Crescenzo et al. (2015), and Vellaisamy and Maheshwari (2014). The processes analyzed here include some processes which have appeared recently in the literature, but not the time-fractional Poisson process (which is a renewal process with nonindependent increments; see Kreer et al. (2014), Kumar et al. (2011), Laskin (2013), and Meerschaert et al. (2011)).

\section{General results}

We now examine in detail the main properties of the process $N^{f}(t), t>0$, with independent increments outlined in the introduction. Our first result is the difference-differential equations governing their state probabilities

$$
p_{k}^{f}(t)=\mathbb{P}\left\{N^{f}(t)=k\right\}, \quad k \geq 0 .
$$

Theorem 2.1. The probabilities $p_{k}^{f}(t)=\mathbb{P}\left\{N^{f}(t)=k\right\}, k \geq 0$, are solutions to the equation

$$
\frac{\mathrm{d}}{\mathrm{d} t} p_{k}^{f}(t)=-f(\lambda) p_{k}^{f}(t)+\sum_{m=1}^{k} \frac{\lambda^{m}}{m !} p_{k-m}^{f}(t) \int_{0}^{\infty} \mathrm{e}^{-s \lambda} s^{m} \nu(\mathrm{d} s), \quad k \geq 0, t>0
$$

with initial condition

$$
p_{k}^{f}(0)= \begin{cases}1, & k=0 \\ 0, & k \geq 1\end{cases}
$$

The PGF $G^{f}(u, t)=\mathbb{E} u^{N^{f}(t)},|u| \leq 1$, satisfies the linear, homogeneous equation

$$
\frac{\partial}{\partial t} G^{f}(u, t)=-f(\lambda(1-u)) G^{f}(u, t), \quad G^{f}(u, 0)=1
$$

and has the form

$$
G^{f}(u, t)=\mathrm{e}^{-t f(\lambda(1-u))} .
$$

Proof. Since $N^{f}(t)$ has independent increments and the distribution of jumps is given by $(1.1)$, we can write

$$
\begin{aligned}
p_{k}^{f}(t+\mathrm{d} t)= & \mathbb{P}\left\{N^{f}[t+\mathrm{d} t)=k\right\} \\
= & \mathbb{P}\left\{\bigcup_{j=0}^{k}\left\{N^{f}(t)=j, N^{f}[t, t+\mathrm{d} t)=k-j\right\}\right\} \\
= & \sum_{j=0}^{k-1} \mathbb{P}\left\{N^{f}(t)=j\right\} \mathrm{d} t \frac{\lambda^{k-j}}{(k-j) !} \int_{0}^{\infty} \mathrm{e}^{-\lambda s} s^{k-j} v(\mathrm{~d} s) \\
& +\mathbb{P}\left\{N^{f}(t)=k\right\}\left(1-\mathrm{d} t \int_{0}^{\infty}\left(1-\mathrm{e}^{-\lambda s}\right) v(\mathrm{~d} s)\right) .
\end{aligned}
$$


A simple expansion permits us to obtain, in the limit, (2.1). From (2.1), we have

$$
\begin{aligned}
\frac{\partial}{\partial t} G^{f}(u, t) & =\sum_{k=0}^{\infty} u^{k} \frac{\mathrm{d}}{\mathrm{d} t} p_{k}^{f}(t) \\
& =-f(\lambda) \sum_{k=0}^{\infty} u^{k} p_{k}^{f}(t)+\sum_{k=1}^{\infty} u^{k} \sum_{m=1}^{k} \frac{\lambda^{m}}{m !} p_{k-m}^{f}(t) \int_{0}^{\infty} \mathrm{e}^{-s \lambda} s^{m} v(\mathrm{~d} s) \\
& =-f(\lambda) G^{f}(u, t)+\sum_{m=1}^{\infty} \frac{\lambda^{m}}{m !} \int_{0}^{\infty} \mathrm{e}^{-s \lambda} s^{m} v(\mathrm{~d} s) \sum_{k=m}^{\infty} u^{k} p_{k-m}^{f}(t) \\
& =-f(\lambda) G^{f}(u, t)+G^{f}(u, t) \int_{0}^{\infty}\left(\mathrm{e}^{-s \lambda(1-u)}-\mathrm{e}^{-s \lambda}\right) v(\mathrm{~d} s) \\
& =-G^{f}(u, t) \int_{0}^{\infty}\left(1-\mathrm{e}^{-s \lambda(1-u)}\right) v(\mathrm{~d} s) \\
& =-G^{f}(u, t) f(\lambda(1-u))
\end{aligned}
$$

In the last step, we take into account the representation (1.2) of the Bernštein functions.

Remark 2.1. The appearance of $p_{k-j}(t), k \geq j \geq 1$, in (2.1) makes the master equation of the state probabilities $p_{k}^{f}(t)$ substantially different from the case of the classical Poisson process. This fact is related to the possibility of jumps of arbitrary height. We also observe that

$$
N^{f}(t) \stackrel{\mathrm{D}}{=} N\left(H^{f}(t)\right),
$$

where $H^{f}$ is the subordinator with Laplace transform (1.3). This can be ascertained by evaluating the PGF of $N\left(H^{f}(t)\right), t>0$, as

$$
\begin{aligned}
\mathbb{E} u^{N\left(H^{f}(t)\right)} & =\sum_{k=0}^{\infty} u^{k} \int_{0}^{\infty} \mathbb{P}\{N(s)=k\} \mathbb{P}\left\{H^{f}(t) \in \mathrm{d} s\right\} \\
& =\int_{0}^{\infty} \mathrm{e}^{-s \lambda(1-u)} \mathbb{P}\left\{H^{f}(t) \in \mathrm{d} s\right\} \\
& =G^{f}(u, t) .
\end{aligned}
$$

In view of (2.3) we can write the distribution of $N^{f}(t)$ as

$$
\begin{aligned}
\mathbb{P}\left\{N^{f}(t)=k\right\} & =\mathbb{P}\left\{N\left(H^{f}(t)\right)=k\right\} \\
& =\int_{0}^{\infty} \mathrm{e}^{-\lambda s} \frac{(\lambda s)^{k}}{k !} \mathbb{P}\left\{H^{f}(t) \in \mathrm{d} s\right\} \\
& =\left.\frac{(-1)^{k}}{k !} \frac{\mathrm{d}^{k}}{\mathrm{~d} u^{k}} \int_{0}^{\infty} \mathrm{e}^{-\lambda s u} \mathbb{P}\left\{H^{f}(t) \in \mathrm{d} s\right\}\right|_{u=1} \\
& =\left.\frac{(-1)^{k}}{k !} \frac{\mathrm{d}^{k}}{\mathrm{~d} u^{k}} \mathrm{e}^{-t f(\lambda u)}\right|_{u=1}, \quad k \geq 0 .
\end{aligned}
$$

Remark 2.2. Equation (2.1) can alternatively be written as

$$
\frac{\mathrm{d}}{\mathrm{d} t} p_{k}^{f}(t)=-f(\lambda(I-B)) p_{k}^{f}(t), \quad t>0, k \geq 0,
$$


where $B$ is the shift operator such that $B p_{k}^{f}(t)=p_{k-1}^{f}(t)$. This can be shown as follows:

$$
\begin{aligned}
-f(\lambda(I-B)) p_{k}^{f}(t) & =-\int_{0}^{\infty}\left(1-\mathrm{e}^{-\lambda s(I-B)}\right) v(\mathrm{~d} s) p_{k}^{f}(t) \\
& =-\int_{0}^{\infty}\left(1-\mathrm{e}^{-\lambda s} \sum_{m=0}^{\infty} \frac{(\lambda s B)^{m}}{m !}\right) v(\mathrm{~d} s) p_{k}^{f}(t) \\
& =-\int_{0}^{\infty}\left(p_{k}^{f}(t)-\mathrm{e}^{-\lambda s} \sum_{m=0}^{k} \frac{(\lambda s)^{m}}{m !} p_{k-m}^{f}(t)\right) v(\mathrm{~d} s) \\
& =-\int_{0}^{\infty}\left(1-\mathrm{e}^{-\lambda s}\right) v(\mathrm{~d} s) p_{k}^{f}(t)+\sum_{m=1}^{k} \frac{\lambda^{m}}{m !} p_{k-m}^{f}(t) \int_{0}^{\infty} \mathrm{e}^{-\lambda s} s^{m} v(\mathrm{~d} s) \\
& =-f(\lambda) p_{k}^{f}(t)+\sum_{m=1}^{k} \frac{\lambda^{m}}{m !} p_{k-m}^{f}(t) \int_{0}^{\infty} \mathrm{e}^{-\lambda s} s^{m} \nu(\mathrm{d} s)
\end{aligned}
$$

Clearly, (2.5) coincides with the right-hand side of (2.1).

A further representation of $N^{f}(t), t>0$, can be obtained as the limit of a suitable compound Poisson process.

\section{Theorem 2.2. Let}

$$
u(n)=\int_{0}^{\infty} \mathbb{P}\{N(s) \geq n\} v(\mathrm{~d} s), \quad n \in \mathbb{N},
$$

where $N(s), s>0$, is a homogeneous Poisson process with rate $\lambda>0$. The compound Poisson process

$$
Z_{n}(t)=\sum_{j=1}^{N((t / \lambda) u(n))} X_{j}, \quad t>0
$$

where $X_{j}, j=1,2, \ldots$, are discrete independent and identically distributed (i.i.d.) random variables $(R V s)$ with probability law

$$
\mathbb{P}\left\{X_{j}=k\right\}=\frac{1}{u(n)} \int_{0}^{\infty} \mathbb{P}\{N(s)=k\} v(\mathrm{~d} s), \quad k \geq n \in \mathbb{N} \text { for all } j=1,2, \ldots,
$$

converges in distribution to the subordinated Poisson process $N^{f}(t)$ as $n \rightarrow 0$. In other words,

$$
N^{f}(t) \stackrel{\mathrm{D}}{=} N\left(H^{f}(t)\right) \stackrel{\mathrm{D}}{=} \lim _{n \rightarrow 0} Z_{n}(t)
$$

Proof. The PGF of $Z_{n}(t)$ can be written as

$$
\begin{aligned}
\mathbb{E} u^{Z_{n}(t)} & =\mathrm{e}^{-t u(n)\left(1-\mathbb{E} u^{X}\right)} \\
& =\exp \left\{-t u(n) \sum_{k=n}^{\infty}\left(1-u^{k}\right) \mathbb{P}\{X=k\}\right\} \\
& =\exp \left\{-t u(n) \sum_{k=n}^{\infty}\left(1-u^{k}\right) \frac{1}{u(n)} \int_{0}^{\infty} \mathbb{P}\{N(s)=k\} v(\mathrm{~d} s)\right\} \\
& =\exp \left\{-t \int_{0}^{\infty} \sum_{k=n}^{\infty}\left(1-u^{k}\right) \mathbb{P}\{N(s)=k\} v(\mathrm{~d} s)\right\}
\end{aligned}
$$


By taking the limit for $n \rightarrow 0$ of (2.7), we have

$$
\begin{aligned}
\lim _{n \rightarrow 0} \mathbb{E} u^{Z_{n}(t)} & =\exp \left\{-t \int_{0}^{\infty} \sum_{k=0}^{\infty}\left(1-u^{k}\right) \mathbb{P}\{N(s)=k\} \nu(\mathrm{d} s)\right\} \\
& =\exp \left\{-t \int_{0}^{\infty}\left(1-\mathrm{e}^{-\lambda s(1-u)}\right) v(\mathrm{~d} s)\right\} \\
& =\mathrm{e}^{-t f(\lambda(1-u))}
\end{aligned}
$$

Remark 2.3. If we take into account processes whose state probabilities satisfy the timefractional equation

$$
\frac{\mathrm{d}^{\nu}}{\mathrm{d} t^{\nu}} p_{k}^{f}(t)=-f(\lambda) p_{k}^{f}(t)+\sum_{m=1}^{k} \frac{\lambda^{m}}{m !} p_{k-m}^{f}(t) \int_{0}^{\infty} \mathrm{e}^{-s \lambda} s^{m} \nu(\mathrm{d} s), \quad k \geq 0, t>0
$$

for $v \in(0,1)$, the corresponding PGF has the form

$$
G_{\nu}^{f}(u, t)=E_{\nu, 1}\left(-t^{\nu} \int_{0}^{\infty}\left(1-\mathrm{e}^{-s \lambda(1-u)}\right) v(\mathrm{~d} s)\right)
$$

where $E_{\nu, 1}(x)$ is the Mittag-Leffler function and the fractional derivative appearing in (2.8) must be understood in the Caputo sense. For the space-fractional Poisson process $f(\lambda)=\lambda^{\alpha}$, $0<\alpha<1$, the distribution of the process related to (2.9) is explicitly given by Orsingher and Polito (2012b, Equation 2.29). The processes whose distribution is governed by (2.8) admits the following representation:

$$
N\left(H^{f}\left(L^{\nu}(t)\right)\right), \quad t>0,
$$

where $L^{v}$ and the stable subordinator $H^{v}$ are related by

$$
\mathbb{P}\left\{L^{\nu}(t)>x\right\}=\mathbb{P}\left\{H^{\nu}(x)<t\right\} .
$$

\section{Hitting-times of the subordinated Poisson process}

In this section we study the hitting-times

$$
T_{k}^{f}=\inf \left\{t \geq 0: N^{f}(t) \geq k\right\}
$$

of the subordinated Poisson processes. The fact that $N^{f}(t)$ performs jumps of random height makes $T_{k}^{f}$ substantially different from the Erlang process related to the homogeneous Poisson process. Indeed, the law of $T_{k}^{f}$ can be written as

$$
\begin{aligned}
\frac{\mathbb{P}\left\{T_{k}^{f} \in \mathrm{d} s\right\}}{\mathrm{d} s} & =\frac{\mathbb{P}\left\{\bigcup_{j=1}^{k}\left\{N^{f}(s)=k-j, N^{f}[s, s+d s) \geq j\right\}\right\}}{\mathrm{d} s} \\
& =\frac{\sum_{j=1}^{k} \mathbb{P}\left\{N^{f}(s)=k-j\right\} \sum_{m=j}^{\infty} \mathbb{P}\left\{N^{f}[s, s+\mathrm{d} s)=m\right\}}{\mathrm{d} s} \\
& =\sum_{j=1}^{k} \int_{0}^{\infty} \mathbb{P}\{N(z)=k-j\} \mathbb{P}\left\{H^{f}(s) \in \mathrm{d} z\right\} \sum_{m=j}^{\infty} \frac{\lambda^{m}}{m !} \int_{0}^{\infty} \mathrm{e}^{-\lambda u} u^{m} v(\mathrm{~d} u)
\end{aligned}
$$




$$
\begin{aligned}
& =\sum_{j=1}^{k} \int_{0}^{\infty} \frac{(\lambda z)^{k-j}}{(k-j) !} \mathrm{e}^{-\lambda z} \mathbb{P}\left\{H^{f}(s) \in \mathrm{d} z\right\} \int_{0}^{\infty} \mathbb{P}\{N(u) \geq j\} \nu(\mathrm{d} u) \\
& =\sum_{j=1}^{k} \frac{(-\lambda)^{k-j}}{(k-j) !} \int_{0}^{\infty} \frac{\mathrm{d}^{k-j}}{\mathrm{~d} \lambda^{k-j}} \mathrm{e}^{-\lambda z} \mathbb{P}\left\{H^{f}(s) \in \mathrm{d} z\right\} \int_{0}^{\infty} \mathbb{P}\{N(u) \geq j\} \nu(\mathrm{d} u) \\
& =\sum_{j=1}^{k} \frac{(-\lambda)^{k-j}}{(k-j) !} \frac{\mathrm{d}^{k-j}}{\mathrm{~d} \lambda^{k-j}} \mathrm{e}^{-s f(\lambda)} \int_{0}^{\infty} \mathbb{P}\{N(u) \geq j\} v(\mathrm{~d} u) \\
& =\sum_{l=0}^{k-1} \frac{(-\lambda)^{l}}{l !}\left(\frac{\mathrm{d}^{l}}{\mathrm{~d} \lambda^{l}} \mathrm{e}^{-s f(\lambda)}\right) \int_{0}^{\infty}\left(1-\sum_{r=0}^{k-l-1} \frac{(\lambda u)^{r}}{r !} \mathrm{e}^{-\lambda u}\right) v(\mathrm{~d} u) .
\end{aligned}
$$

The distribution of $T_{k}^{f}$ can also be obtained by observing that

$$
\mathbb{P}\left\{T_{k}^{f}<s\right\}=\mathbb{P}\left\{N^{f}(s) \geq k\right\}=\sum_{j=k}^{\infty} \int_{0}^{\infty} \mathrm{e}^{-\lambda z} \frac{(\lambda z)^{j}}{j !} \mathbb{P}\left\{H^{f}(s) \in \mathrm{d} z\right\}
$$

and, thus,

$$
\begin{aligned}
\frac{\mathbb{P}\left\{T_{k}^{f} \in \mathrm{d} s\right\}}{\mathrm{d} s} & =\frac{\mathrm{d}}{\mathrm{d} s} \sum_{j=k}^{\infty} \int_{0}^{\infty} \mathrm{e}^{-\lambda z} \frac{(\lambda z)^{j}}{j !} \mathbb{P}\left\{H^{f}(s) \in \mathrm{d} z\right\} \\
& =\frac{\mathrm{d}}{\mathrm{d} s} \int_{0}^{\infty} \mathbb{P}\{N(z) \geq k\} \mathbb{P}\left\{H^{f}(s) \in \mathrm{d} z\right\} \\
& =\frac{\mathrm{d}}{\mathrm{d} s} \int_{0}^{\infty}\left(1-\sum_{l=0}^{k-1} \frac{(\lambda z)^{l}}{l !} \mathrm{e}^{-\lambda z}\right) \mathbb{P}\left\{H^{f}(s) \in \mathrm{d} z\right\} \\
& =-\frac{\mathrm{d}}{\mathrm{d} s} \sum_{l=0}^{k-1} \frac{(-\lambda)^{l}}{l !} \int_{0}^{\infty} \frac{\mathrm{d}^{l}}{\mathrm{~d} \lambda^{l}} \mathrm{e}^{-\lambda z} \mathbb{P}\left\{H^{f}(s) \in \mathrm{d} z\right\} \\
& =-\frac{\mathrm{d}}{\mathrm{d} s} \sum_{l=0}^{k-1} \frac{(-\lambda)^{l}}{l !} \frac{\mathrm{d}^{l}}{\mathrm{~d} \lambda^{l}} \mathrm{e}^{-s f(\lambda)}, \quad s>0 .
\end{aligned}
$$

For $f(\lambda)=\lambda$ from (3.2), we extract the Erlang distribution for the first-passage time of the Poisson process.

Remark 3.1. In particular, we observe that, from (3.1) and (3.2), we have

$$
\mathbb{P}\left\{T_{1}^{f} \in \mathrm{d} s\right\}=f(\lambda) \mathrm{e}^{-s f(\lambda)} \mathrm{d} s, \quad s>0 .
$$

This proves that the waiting time of the first event for all subordinated Poisson processes is exponential. Instead,

$$
\mathbb{P}\left\{T_{2}^{f} \in \mathrm{d} s\right\}=\mathrm{e}^{-s f(\lambda)}\left(f(\lambda)-\lambda f^{\prime}(\lambda)+\lambda s f^{\prime}(\lambda) f(\lambda)\right) \mathrm{d} s, \quad s>0,
$$

and for $f(\lambda)=\lambda$ (ordinary Poisson case) we recover the gamma distribution with parameters $(2, \lambda)$. Equation (3.4) can also be obtained from (3.1). For $f(\lambda)=\lambda^{\alpha}$ (space-fractional Poisson process), we have

$$
\mathbb{P}\left\{T_{2}^{\alpha} \in \mathrm{d} s\right\}=\mathrm{d} s \lambda^{\alpha} \mathrm{e}^{-s \lambda^{\alpha}}\left(1-\alpha+\alpha s \lambda^{\alpha}\right), \quad s>0 .
$$


Clearly, (3.4) cannot be the distribution of the sum of exponential RVs (3.3) because the second event can also be obtained as a jump of magnitude equal to 2 . Finally, we observe that

$$
\mathbb{P}\left\{T_{k}^{f} \in \mathrm{d} s\right\}=\mathbb{P}\left\{T_{k-1}^{f} \in \mathrm{d} s\right\}-\frac{(-\lambda)^{k-1}}{(k-1) !} \frac{\mathrm{d}}{\mathrm{d} s} \frac{\mathrm{d}^{k-1}}{\mathrm{~d} \lambda^{k-1}} \mathrm{e}^{-s f(\lambda)} \mathrm{d} s, \quad s \in(0, \infty),
$$

so that the distributions of $T_{k}^{f}$ can be derived successively.

Here we derive the equation governing the distribution of $T_{k}^{f}$. First, we note that

$$
\mathfrak{G}^{f}(u, s)=\sum_{k=1}^{\infty} u^{k} \frac{\mathbb{P}\left\{T_{k}^{f} \in \mathrm{d} s\right\}}{\mathrm{d} s}=\frac{u}{1-u} f(\lambda(1-u)) \mathrm{e}^{-s f(\lambda(1-u))}, \quad s>0,|u|<1 .
$$

This can be proved as follows:

$$
\begin{aligned}
\mathfrak{G}^{f}(u, s) & =\sum_{k=1}^{\infty} u^{k} \frac{\mathbb{P}\left\{T_{k}^{f} \in \mathrm{d} s\right\}}{\mathrm{d} s} \\
& =\frac{\mathrm{d}}{\mathrm{d} s} \sum_{j=1}^{\infty} \sum_{k=1}^{j} u^{k} \int_{0}^{\infty} \mathrm{e}^{-\lambda z} \frac{(\lambda z)^{j}}{j !} \mathbb{P}\left\{H^{f}(s) \in \mathrm{d} z\right\} \\
& =\frac{\mathrm{d}}{\mathrm{d} s} \sum_{j=1}^{\infty} \frac{u^{j+1}-u}{u-1} \int_{0}^{\infty} \mathrm{e}^{-\lambda z} \frac{(\lambda z)^{j}}{j !} \mathbb{P}\left\{H^{f}(s) \in \mathrm{d} z\right\} \\
& =\frac{\mathrm{d}}{\mathrm{d} s} \int_{0}^{\infty} \frac{u}{u-1}\left(\mathrm{e}^{-\lambda z(1-u)}-1\right) \mathbb{P}\left\{H^{f}(s) \in \mathrm{d} z\right\} \\
& =\frac{u}{1-u} f(\lambda(1-u)) \mathrm{e}^{-s f(\lambda(1-u))} .
\end{aligned}
$$

Theorem 3.1. The probability density $q_{k}^{f}(t)=\mathbb{P}\left\{T_{k}^{f} \in \mathrm{d} t\right\} / \mathrm{d} t$ solves the following equation:

$$
f(\lambda(I-B)) q_{k}^{f}(t)=-\frac{\mathrm{d}}{\mathrm{d} t} q_{k}^{f}(t) .
$$

Proof. Because

$$
f(\lambda(I-B)) q_{k}^{f}(t)=f(\lambda) q_{k}^{f}(t)-\sum_{m=1}^{k-1} \int_{0}^{\infty} \mathrm{e}^{-\lambda s} \frac{(\lambda s)^{m}}{m !} v(\mathrm{~d} s) q_{k-m}^{f}(t)
$$

we can write, since $q_{0}(t)=0$, for $t>0$,

$$
\begin{aligned}
\sum_{k=1}^{\infty} u^{k} f(\lambda(I-B)) q_{k}^{f}(t) & =f(\lambda) \mathfrak{G}^{f}(u, t)-\sum_{k=1}^{\infty} u^{k} \sum_{m=1}^{k} \int_{0}^{\infty} \mathrm{e}^{-\lambda s} \frac{(\lambda s)^{m}}{m !} v(\mathrm{~d} s) q_{k-m}^{f}(t) \\
& =f(\lambda) \mathfrak{G}^{f}(u, t)-\sum_{m=1}^{\infty} \sum_{k=m}^{\infty}\left(\int_{0}^{\infty} \mathrm{e}^{-\lambda s} \frac{(\lambda s)^{m}}{m !} v(\mathrm{~d} s)\right) u^{k} q_{k-m}^{f}(t) \\
& =f(\lambda) \mathfrak{G}^{f}(u, t)-\mathfrak{G}^{f}(u, t) \int_{0}^{\infty} \mathrm{e}^{-\lambda s}\left(\mathrm{e}^{u \lambda s}-1\right) v(\mathrm{~d} s) \\
& =f(\lambda(1-u)) \mathfrak{G}^{f}(u, t) .
\end{aligned}
$$


From (3.5), we obtain

$$
f(\lambda(1-u)) \mathfrak{G}^{f}(u, t)=-\frac{\mathrm{d}}{\mathrm{d} t} \mathfrak{G}^{f}(u, t),
$$

which completes the proof.

\section{Some particular cases}

In this section we specialize the function $f$ in order to analyze some particular cases of $N^{f}(t), t>0$.

\subsection{The space-fractional Poisson process}

If

$$
v(\mathrm{~d} s)=\frac{\alpha s^{-\alpha-1}}{\Gamma(1-\alpha)} \mathrm{d} s, \quad \alpha \in(0,1),
$$

we obtain the space-fractional Poisson process $N^{\alpha}(t), t>0$, studied in Orsingher and Polito (2012b). The distributions of jumps (1.1) and (1.2) specialize to

$$
\begin{array}{rlrl}
\mathbb{P}\left\{N^{\alpha}[t, t+\mathrm{d} t)=k\right\} & = \begin{cases}\frac{(-1)^{k+1} \lambda^{\alpha}}{k !} \alpha(\alpha-1) \cdots(\alpha-k+1) \mathrm{d} t+o(\mathrm{~d} t), & k>0, \\
1-\lambda^{\alpha} \mathrm{d} t+o(\mathrm{~d} t), & k=0,\end{cases} \\
& =\lambda^{\alpha} \mathrm{d} t \frac{(-1)^{k+1} \Gamma(\alpha+1)}{k ! \Gamma(\alpha+1-k)}, \quad k>0 &
\end{array}
$$

since $f(\lambda)=\lambda^{\alpha}$. The distribution of $N^{\alpha}$ can be written in three different ways as

$$
\begin{aligned}
p_{k}^{\alpha}(t) & =\mathbb{P}\left\{N^{\alpha}(t)=k\right\} \\
& =\frac{(-1)^{k}}{k !} \sum_{r=0}^{\infty} \frac{\left(-\lambda^{\alpha} t\right)^{r}}{r !} \frac{\Gamma(\alpha r+1)}{\Gamma(\alpha r+1-k)} \\
& =\frac{(-1)^{k}}{k !} \sum_{r=0}^{\infty} \frac{\left(-\lambda^{\alpha} t\right)^{r}}{r !}(\alpha r)(\alpha r-1) \cdots(\alpha r-k+1) \\
& =\left.\frac{(-1)^{k}}{k !} \frac{\mathrm{d}^{k}}{\mathrm{~d} u^{k}} \mathrm{e}^{-t \lambda^{\alpha} u^{\alpha}}\right|_{u=1}
\end{aligned}
$$

and we note that the probabilities (4.1) can be obtained directly from (4.2).

Remark 4.1. In light of (4.2) the distribution of the space-fractional Poisson process has the following alternative form:

$$
p_{k}^{\alpha}(t)=\frac{\mathrm{e}^{-\lambda^{\alpha} t}}{k !}\left[c_{k, k} t^{k}+c_{k-1, k} t^{k-1}+\cdots+c_{2, k} t^{2}+c_{1, k} t\right],
$$

where the coefficients $c_{j, k}, j=1, \ldots, k$, can be computed by means of successive derivatives. In particular, we have

$$
\begin{aligned}
c_{k, k} & =\left(\alpha \lambda^{\alpha}\right)^{k}, & c_{k-1, k} & =\alpha^{k-1}(1-\alpha) \frac{k(k-1)}{2}\left(\lambda^{\alpha}\right)^{k-1}, \\
c_{2, k} & =\left(\lambda^{\alpha}\right)^{2} \alpha^{2} \prod_{j=1}^{k-2}(j-\alpha) \frac{k(k-1)}{2}, & c_{1, k} & =\alpha \lambda^{\alpha} \prod_{j=1}^{k-1}(j-\alpha) .
\end{aligned}
$$


For $\alpha=1$ all the coefficients $c_{j, k}, j=1, \ldots, k-1$, are equal to 0 and from (4.3) we recover the distribution of the homogeneous Poisson process. The coefficients (4.4) are sufficient to obtain $p_{j}^{\alpha}(t), 1 \leq j \leq 4$ as

$$
\begin{aligned}
& p_{2}^{\alpha}(t)= \frac{\mathrm{e}^{-\lambda^{\alpha} t}}{2}\left[\left(\lambda^{\alpha} \alpha t\right)^{2}+\alpha(1-\alpha) \lambda^{\alpha} t\right] \\
& p_{3}^{\alpha}(t)= \frac{\mathrm{e}^{-\lambda^{\alpha} t}}{3 !}\left[\left(\lambda^{\alpha} \alpha t\right)^{3}+3\left(\lambda^{\alpha} \alpha t\right)^{2}(1-\alpha)+\left(\lambda^{\alpha} \alpha t\right)(1-\alpha)(2-\alpha)\right], \\
& p_{4}^{\alpha}(t)=\frac{\mathrm{e}^{-\lambda^{\alpha} t}}{4 !}\left[\left(\alpha \lambda^{\alpha} t\right)^{4}+6\left(\lambda^{\alpha} \alpha t\right)^{3}(1-\alpha)+6\left(\alpha \lambda^{\alpha} t\right)^{2}(1-\alpha)(2-\alpha)\right. \\
&\left.\quad+\lambda^{\alpha} \alpha t(1-\alpha)(2-\alpha)(3-\alpha)\right] .
\end{aligned}
$$

Remark 4.2. In light of the independence of increments for the space-fractional Poisson process, we have, for $0 \leq r \leq k$ and $0 \leq s \leq t$,

$$
\begin{aligned}
\mathbb{P}\left\{N^{\alpha}(s)=r \mid N^{\alpha}(t)=k\right\} & =\frac{\mathbb{P}\left\{N^{\alpha}(s)=r\right\} \mathbb{P}\left\{N^{\alpha}(t-s)=k-r\right\}}{\mathbb{P}\left\{N^{\alpha}(t)=k\right\}} \\
& =\left.\left(\begin{array}{l}
k \\
r
\end{array}\right) \frac{\left(\mathrm{d}^{r} / \mathrm{d} u^{r}\right) \mathrm{e}^{-s \lambda^{\alpha} u^{\alpha}}\left(\mathrm{d}^{k-r} / \mathrm{d} u^{k-r}\right) \mathrm{e}^{-(t-s) \lambda^{\alpha} u^{\alpha}}}{\left(\mathrm{d}^{k} / \mathrm{d} u^{k}\right) \mathrm{e}^{-\lambda^{\alpha} t u^{\alpha}}}\right|_{u=1} \\
& =\left(\begin{array}{l}
k \\
r
\end{array}\right) \frac{\sum_{j=1}^{r} c_{j, r} s^{j} \sum_{n=1}^{k-r} c_{n, k-r}(t-s)^{n}}{\sum_{l=1}^{k} c_{l, k} t^{l}},
\end{aligned}
$$

where we used (4.3). For $\alpha=1$, we obtain $c_{r, r}, c_{k-r, k-r}, c_{k, k} \neq 0$ and $c_{j, r}=c_{n, k-r}=c_{l, k}=0$ for $j<r, n<k-r, l<k$ and, thus, from (4.5) we recover the binomial distribution.

In the time interval $[0, t]$ the instants of occurrences of the upward jumps are denoted by $\tau_{j}^{l_{j}}, 1 \leq j \leq r, l_{j} \geq 1$, where $r$ is the number of jumps in $[0, t]$ and $l_{j}$ is the height of the $j$ th jump. We can write the following distribution for $r \leq k$ :

$$
\begin{aligned}
& \mathbb{P}\left\{\bigcap_{j=1}^{r}\left\{\tau_{j}^{l_{j}} \in \mathrm{d} t_{j}\right\} \mid N^{\alpha}(t)=k\right\} \\
& \quad=\frac{k !\left(\lambda^{\alpha} \Gamma(\alpha+1)\right)^{r}(-1)^{k+r} \prod_{j=1}^{r} \mathrm{~d} t_{j} / l_{j} ! \Gamma\left(\alpha+1-l_{j}\right)}{\sum_{n=1}^{k} c_{n, k} t^{n}} \text { for } 0<t_{1}<\cdots<t_{r}<t .
\end{aligned}
$$

The distribution (4.6) can be evaluated by considering

$$
\begin{aligned}
& \mathbb{P}\left\{\bigcap_{j=1}^{r}\left\{\tau_{j}^{l_{j}} \in \mathrm{d} t_{j}\right\} \mid N^{\alpha}(t)=k\right\} \\
& \quad=\frac{1}{\mathbb{P}\left\{N^{\alpha}(t)=k\right\}} \mathbb{P}\left\{\bigcap_{j=1}^{r+1}\left\{N^{\alpha}\left[t_{j-1}, t_{j}\right)=0, N^{\alpha}\left[t_{j}, t_{j}+\mathrm{d} t_{j}\right)=l_{j}\right\}\right\},
\end{aligned}
$$

where $t_{0}=0$ and $t_{r+1}=t$. Since the space-fractional Poisson process has independent increments and in view of the transition probabilities (4.1), we arrive at (4.6). If $N^{\alpha}(t)=k$, 
and $l_{j}=1$, for all $j$, we have

$$
\mathbb{P}\left\{\bigcap_{j=1}^{k}\left\{\tau_{j}^{1} \in \mathrm{d} t_{j}\right\} \mid N^{\alpha}(t)=k\right\}=\frac{k !\left(\alpha \lambda^{\alpha}\right)^{k}}{\sum_{j=1}^{k} c_{j, k} t^{j}} \prod_{j=1}^{k} \mathrm{~d} t_{j}
$$

on the simplex $S_{t}=\left\{t_{i}, i=1, \ldots, k: 0<t_{1}<t_{2}<\cdots<t_{k}<t\right\}$. Clearly, for $\alpha=1$, from (4.7) we retrieve the uniform distribution on the set $S_{t}$. Since the coefficients $c_{j, k}$ can be calculated in some specific cases, the distribution can be written down explicitly for small values of $k$. For example, for $k=2$, we have

$$
\begin{array}{cl}
\mathbb{P}\left\{\bigcap_{j=1}^{2}\left\{\tau_{j}^{1} \in \mathrm{d} t_{j}\right\} \mid N^{\alpha}(t)=2\right\}=\frac{\left(\alpha \lambda^{\alpha}\right)^{2} \mathrm{~d} t_{1} \mathrm{~d} t_{2}}{\left(\alpha \lambda^{\alpha} t\right)^{2}+\alpha(1-\alpha) \lambda^{\alpha} t}, & 0<t_{1}<t_{2}<t, \\
\mathbb{P}\left\{\tau_{1}^{2} \in \mathrm{d} t_{1} \mid N^{\alpha}(t)=2\right\}=\frac{(1 / 2) \alpha(1-\alpha) \lambda^{\alpha} \mathrm{d} t_{1}}{\left(\alpha \lambda^{\alpha} t\right)^{2}+\alpha(1-\alpha) \lambda^{\alpha} t}, & 0<t_{1}<t .
\end{array}
$$

\subsection{Poisson process with a relativistic (tempered) stable subordinator}

In the case where the Lévy measure has the form

$$
\nu(\mathrm{d} s)=\frac{\alpha s^{-\alpha-1} \mathrm{e}^{-\theta s}}{\Gamma(1-\alpha)} \mathrm{d} s, \quad \theta>0,0<\alpha<1,
$$

we obtain an extension of the space-fractional Poisson process. This new Poisson process has the form $N^{\alpha, \theta}(t) \stackrel{\mathrm{D}}{=} N\left(H^{\alpha, \theta}(t)\right)$, where $H^{\alpha, \theta}$ is the relativistic or tempered stable subordinator. Such a process is called relativistic since it appeared in the study of the stability of relativistic matter (see Lieb (1990)). From (2.2), we obtain the PGF as

$$
\begin{aligned}
G^{\alpha, \theta}(u, t) & =\exp \left\{-t \int_{0}^{\infty}\left(1-\mathrm{e}^{-\lambda(1-u) s}\right) \frac{\alpha s^{-\alpha-1} \mathrm{e}^{-\theta s}}{\Gamma(1-\alpha)} \mathrm{d} s\right\} \\
& =\mathrm{e}^{-t\left\{[\theta+\lambda(1-u)]^{\alpha}-\theta^{\alpha}\right\}} \\
& =\mathrm{e}^{\theta^{\alpha} t} \sum_{k=0}^{\infty} \frac{[-t(\theta+\lambda(1-u))]^{\alpha}}{k !} \\
& =\mathrm{e}^{\theta^{\alpha} t} \sum_{k=0}^{\infty} \frac{\left[-t(\theta+\lambda)^{\alpha}\right]^{k}}{k !}\left(1-\frac{\lambda u}{\theta+\lambda}\right)^{\alpha k} \\
& =\mathrm{e}^{\theta^{\alpha} t} \sum_{k=0}^{\infty} \frac{(-t(\theta+\lambda))^{k}}{k !} \sum_{m=0}^{\infty} \frac{\Gamma(\alpha k+1)}{\Gamma(\alpha k+1-m) m !}\left(-\frac{\lambda u}{\theta+\lambda}\right)^{m} \\
& =\sum_{m=0}^{\infty} u^{m}\left[\frac{(-1)^{m}}{m !} \frac{\lambda^{m} \mathrm{e}^{\theta^{\alpha} t}}{(\theta+\lambda)^{m}} \sum_{k=0}^{\infty} \frac{\left[-t(\theta+\lambda)^{\alpha}\right]^{k}}{k !} \frac{\Gamma(\alpha k+1)}{\Gamma(\alpha k+1-m)}\right] .
\end{aligned}
$$

From (4.8) we extract the distribution of $N^{\alpha, \theta}(t), t>0$, as

$$
\mathbb{P}\left\{N^{\alpha, \theta}(t)=m\right\}=\frac{(-1)^{m}}{m !} \frac{\lambda^{m} \mathrm{e}^{\theta^{\alpha} t}}{(\theta+\lambda)^{m}} \sum_{k=0}^{\infty} \frac{\left(-t(\lambda+\theta)^{\alpha}\right)^{k}}{k !} \frac{\Gamma(\alpha k+1)}{\Gamma(\alpha k+1-m)}, \quad m \geq 0
$$


For $\theta=0$, (4.9) yields the distribution of the space-fractional Poisson process (see Orsingher and Polito (2012b, Equation 1.2)). An alternative form of (4.9) can be written as

$$
\mathbb{P}\left\{N^{\alpha, \theta}(t)=m\right\}=\left.\frac{(-1)^{m}}{m !}\left(\frac{\lambda}{\lambda+\theta}\right)^{m} \mathrm{e}^{\theta^{\alpha} t} \frac{\mathrm{d}^{m}}{\mathrm{~d} u^{m}} \mathrm{e}^{-t u^{\alpha}(\theta+\lambda)^{\alpha}}\right|_{u=1}
$$

and can be derived either from (4.9) or from (2.4). From (4.10) (and also from (1.1)), we have, for $m \geq 1$,

$$
\begin{aligned}
& \mathbb{P}\left\{N^{\alpha, \theta}[t, t+\mathrm{d} t)=m\right\} \\
& \quad=\frac{(-1)^{m+1}(\lambda /(\lambda+\theta))^{m}}{m !}(\lambda+\theta)^{\alpha} \alpha(\alpha-1) \cdots(\alpha-m+1) \mathrm{d} t
\end{aligned}
$$

and this represents the distribution of the jumps during $[t, t+\mathrm{d} t)$. From (4.11) we see that high jumps have less probability of occurring than in the space-fractional Poisson process.

Remark 4.3. We note that

$$
\begin{gathered}
\mathbb{E} N^{\alpha, \theta}(t)=\alpha \lambda \theta^{\alpha-1} t, \quad \operatorname{var}\left[N^{\alpha, \theta}(t)\right]=\alpha \lambda \theta^{\alpha-2}(\lambda(1-\alpha)+\theta) t \\
\operatorname{cov}\left[N^{\alpha, \theta}(t) N^{\alpha, \theta}(s)\right]=\alpha \lambda \theta^{\alpha-2}(\lambda(1-\alpha)+\theta)(s \wedge t)
\end{gathered}
$$

From (4.12) and (4.13), it is apparent that in the space-fractional Poisson process $(\theta=0)$ the mean values diverge.

\subsection{Poisson process with gamma subordinator}

For the Lévy measure

$$
v(\mathrm{~d} s)=\frac{\mathrm{e}^{-s}}{s} \mathrm{~d} s, \quad s>0,
$$

the distribution of the related Poisson process has a particularly simple and interesting form, since it is the negative binomial. We note that the Bernštein function corresponding to the Lévy measure $v(\mathrm{~d} s)=\left(\mathrm{e}^{-s} / s\right) \mathrm{d} s$ is

$$
f(x)=\int_{0}^{\infty}\left(1-\mathrm{e}^{-s x}\right) \frac{\mathrm{e}^{-s}}{s} \mathrm{~d} s=\log (1+x) .
$$

Therefore, the PGF (2.2) reduces to the form

$$
G^{\Gamma}(u, t)=\mathrm{e}^{-t \log (1+\lambda(1-u))}=(1+\lambda(1-u))^{-t}
$$

and, thus, the intertime $T$ between successive clusters of events has law

$$
\mathbb{P}\{T>t\}=\frac{1}{(1+\lambda)^{t}}
$$

Equation (4.14) is clearly the PGF of $N^{\Gamma}(t) \stackrel{\mathrm{D}}{=} N\left(H^{\Gamma}(t)\right)$, where $H^{\Gamma}$ is the gamma subordinator with Laplace transform

$$
\mathbb{E e}^{-\mu H^{f}(t)}=(1+\mu)^{-t} .
$$

The distribution of $N^{\Gamma}(t), t>0$, can be extracted from (4.14). 
Proposition 4.1. The process $N^{\Gamma}(t), t>0$, has the following distribution:

$$
\begin{array}{rlrl}
\mathbb{P}\left\{N^{\Gamma}(t)=k\right\} & = \begin{cases}\frac{\lambda^{k} t(t+1) \cdots(t+k-1)}{(\lambda !} \frac{1}{(\lambda+1)^{t+k}}, & k \geq 1, \\
\frac{1}{(1+\lambda)^{t}}, & k=0,\end{cases} \\
& =\frac{\lambda^{k} \Gamma(k+t)}{\Gamma(t) k !(\lambda+1)^{t+k}}, \quad k \geq 0 . &
\end{array}
$$

Proof. The distribution of $N\left(H^{\Gamma}(t)\right), t>0$, is the negative binomial (see, for example, Kozubowski and Podgórski (2009)). Its PGF is

$$
\begin{aligned}
G^{\Gamma}(u, t) & =(1+\lambda(1-u))^{-t} \\
& =\left(1-\frac{\lambda u}{1+\lambda}\right)^{-t}(1+\lambda)^{-t} \\
& =(1+\lambda)^{-t} \sum_{k=0}^{\infty} \frac{\Gamma(-t+1)}{k ! \Gamma(-t+1-k)}\left(-\frac{\lambda u}{1+\lambda}\right)^{k} \\
& =\sum_{k=0}^{\infty} u^{k}\left[\frac{\lambda^{k} \Gamma(t+k)}{k ! \Gamma(t)} \frac{1}{(1+\lambda)^{t+k}}\right]
\end{aligned}
$$

Remark 4.4. The distribution (4.15) of $N^{\Gamma}(t)$ is written as

$$
\mathbb{P}\left\{N^{\Gamma}(t)=k\right\}=\frac{\lambda^{k}}{(1+\lambda)^{k+t}} \frac{\Gamma(k+t)}{\Gamma(t)} \frac{1}{k !}=\mathbb{E} \mathbb{P}\{N(\mathcal{T})=k\},
$$

where $\mathcal{T}$ is gamma distributed with parameters $(1, t)$ (that is the distribution of $H^{\Gamma}$ ) and $N$ is a homogeneous Poisson process with parameter $\lambda$, independent from $\mathcal{T}$. Furthermore, (4.15) can be regarded as an extension of the negative binomial $\mathscr{B}^{i}$, where

$$
\mathbb{P}\left\{\mathscr{B}^{i}=k\right\}=\frac{\Gamma(i+k)}{\Gamma(i) \Gamma(k+1)} p^{i} q^{k}
$$

for $i=t, p=1 /(1+\lambda), q=\lambda /(1+\lambda)$ (see also Kozubowski and Podgórski (2009)).

Corollary 4.1. The distribution of jumps in this case has the form

$$
\mathbb{P}\left\{N^{\Gamma}[t, t+\mathrm{d} t)=k\right\}= \begin{cases}\left(\frac{\lambda}{\lambda+1}\right)^{k} \frac{1}{k} \mathrm{~d} t, & k \geq 1, \\ 1-\log (1+\lambda) \mathrm{d} t, & k=0,\end{cases}
$$

as can be inferred from (1.1) and also from (4.15). The jumps follow logarithmic distribution.

Remark 4.5. We observe that, for $s<t, r \leq k$,

$$
\begin{aligned}
\mathbb{P}\left\{N^{\Gamma}(s)=r \mid N^{\Gamma}(t)=k\right\} & =\left(\begin{array}{l}
k \\
r
\end{array}\right) \frac{\Gamma(t)}{\Gamma(t-s) \Gamma(s)} \frac{\Gamma(s+r) \Gamma(t-s+k-r)}{\Gamma(k+t)} \\
& =\left(\begin{array}{l}
k \\
r
\end{array}\right) \frac{B(s+r, t-s+k-r)}{B(s, t-s)} .
\end{aligned}
$$


Furthermore, from (4.17) we can write, for $0 \leq r \leq k$,

$$
\begin{aligned}
\mathbb{P}\left\{N^{\Gamma}(s)=r \mid N^{\Gamma}(t)=k\right\} & =\left(\begin{array}{l}
k \\
r
\end{array}\right) \frac{\int_{0}^{1} x^{s+r-1}(1-x)^{t-s+k-r-1} \mathrm{~d} x}{B(s, t-s)} \\
& =\mathbb{E}\left[\left(\begin{array}{l}
k \\
r
\end{array}\right) X^{r}(1-X)^{k-r}\right],
\end{aligned}
$$

where $X$ is an RV with beta distribution with parameters $s$ and $t-s$, that is

$$
\mathbb{P}\{X \in \mathrm{d} x\}=\frac{x^{s-1}(1-x)^{t-s-1}}{B(s, t-s)} \mathrm{d} x .
$$

Equation (4.18) shows that in the gamma-Poisson process the conditional number of events at time $s<t$ is a randomized binomial if $N(t)=k$.

Remark 4.6. In view of (4.15), (4.16), and of the independence of the increments of the gamma-Poisson process, we have

$$
\mathbb{P}\left\{\bigcap_{j=1}^{r}\left\{\tau_{j}^{l_{j}} \in \mathrm{d} t_{j}\right\} \mid N^{\Gamma}(t)=k\right\}=\frac{k ! \Gamma(t)}{\Gamma(t+k)} \prod_{j=1}^{r} \frac{\mathrm{d} t_{j}}{l_{j}}
$$

on the simplex $0<t_{1}<t_{2}<\cdots<t_{r}<t$ and $\sum_{j=1}^{r} l_{j}=k$. Some special cases of (4.19) are

(i) $l_{j}=1$, for all $j=1, \ldots, r$ and, thus, $r=k$. In this case, we have

$$
\mathbb{P}\left\{\bigcap_{j=1}^{k}\left\{\tau_{j}^{1} \in \mathrm{d} t_{1}\right\} \mid N^{\Gamma}(t)=k\right\}=\frac{k ! \Gamma(t)}{\Gamma(t+k)} \prod_{j=1}^{k} \mathrm{~d} t_{j}, \quad 0<t_{1}<\cdots<t_{k}<t
$$

(ii) $l_{1}=k$ and, thus, $r=1$ (unique jump of height $k$ ). Here, we obtain

$$
\mathbb{P}\left\{\tau_{1}^{k} \in \mathrm{d} t_{1} \mid N^{\Gamma}(t)=k\right\}=\frac{\mathrm{d} t_{1}}{k} \frac{k ! \Gamma(t)}{\Gamma(t+k)}, \quad 0<t_{1}<t ;
$$

(iii) $k=2 m, l_{j}=2$, for all $j$ and, therefore, $r=m$. We have

$$
\mathbb{P}\left\{\bigcap_{j=1}^{m}\left\{\tau_{j}^{2} \in \mathrm{d} t_{j}\right\} \mid N^{\Gamma}(t)=2 m\right\}=\frac{(2 m) ! \Gamma(t)}{2^{m} \Gamma(t+2 m)} \prod_{j=1}^{m} \mathrm{~d} t_{j}
$$

for $0<t_{1}<\cdots<t_{m}<t$.

Remark 4.7. From (4.14) we obtain the $r$ th factorial moment of $N^{\Gamma}(t), t>0$, as

$$
\mathbb{E}\left[N^{\Gamma}(t)\left(N^{\Gamma}(t)-1\right) \cdots\left(N^{\Gamma}(t)-r+1\right)\right]=\lambda^{r} t(t+1) \cdots(t+r-1) .
$$

When $\mathbb{E} N^{\Gamma}(t)=\lambda t$, the variance becomes var $N^{\Gamma}(t)=\lambda t(\lambda+1)$ and

$$
\operatorname{cov}\left[N^{\Gamma}(t), N^{\Gamma}(s)\right]=\lambda(\lambda+1)(s \wedge t)
$$

Furthermore, we have

$$
\mathbb{E}\left[\int_{0}^{t} N^{\Gamma}(s) \mathrm{d} s\right]=\frac{\lambda t^{2}}{2}, \quad \operatorname{var}\left[\int_{0}^{t} N^{\Gamma}(s) \mathrm{d} s\right]=\frac{\lambda(\lambda+1) t^{3}}{3} .
$$


Remark 4.8. We can also write the following conditional mean values:

$$
\begin{gathered}
\mathbb{E}\left[N^{\Gamma}(s) \mid N^{\Gamma}(t)=k\right]=\frac{k s}{t}, \quad 0<s<t, \\
\mathbb{E}\left[N^{\Gamma}(s) N^{\Gamma}(w) \mid N^{\Gamma}(t)=k\right] \\
=\frac{k s}{t}+k(k-1) \frac{s(s+1)}{t(t+1)}+k(k-1) \frac{s(w-s)}{t(t+1)} \quad \text { for } 0<s<w<t, \\
\operatorname{cov}\left[N^{\Gamma}(s), N^{\Gamma}(w) \mid N^{\Gamma}(t)=k\right]=\frac{k}{t(t+1)}\left(1+\frac{k}{t}\right) \min (s, w) \min (t-s, t-w) .
\end{gathered}
$$

As a special case, from (4.20) we extract the conditional variance as

$$
\operatorname{var}\left[N^{\Gamma}(s) \mid N^{\Gamma}(t)=k\right]=\frac{s k(t-s)}{t(t+1)}\left(1+\frac{k}{t}\right), \quad 0<s<t,
$$

and from (4.8),

$$
\mathbb{E}\left[\left(N^{\Gamma}(s)\right)^{2} \mid N^{\Gamma}(t)=k\right]=\frac{s}{t} k+k(k-1)\left(\frac{s}{t}\right) \frac{s+1}{t+1} .
$$

As a check, we observe that

$$
\begin{aligned}
\operatorname{var} N^{\Gamma}(s) & =\mathbb{E}\left[\operatorname{var}\left[N^{\Gamma}(s) \mid N^{\Gamma}(t)\right]\right]+\operatorname{var}\left[\mathbb{E}\left[N^{\Gamma}(s) \mid N^{\Gamma}(t)\right]\right] \\
& =\frac{s(t-s)}{t(t+1)} \mathbb{E} N^{\Gamma}(t)+\frac{s(t-s)}{t^{2}(t+1)} \mathbb{E}\left(N^{\Gamma}(t)\right)^{2}+\frac{s^{2}}{t^{2}} \operatorname{var} N^{\Gamma}(t) \\
& =\left(\frac{s}{t}\right) \frac{t-s}{t+1} \lambda t+\left(\frac{s}{t^{2}}\right) \frac{t-s}{t+1}\left(\lambda(\lambda+1) t+\lambda^{2} t^{2}\right)+\frac{s^{2}}{t^{2}} \lambda(\lambda+1) t \\
& =\lambda(\lambda+1) s .
\end{aligned}
$$

Remark 4.9. We consider here the distribution of $N_{1}^{\Gamma}(t)-N_{2}^{\Gamma}(t), t>0$, where $N_{j}^{\Gamma}, j=1,2$, are independent gamma-Poisson processes. This leads to a generalization of the Skellam law of the difference of independent homogeneous Poisson processes. We have

$$
\begin{aligned}
& \mathbb{P}\left\{N_{1}^{\Gamma}(t)-N_{2}^{\Gamma}(t)=r\right\} \\
& =\sum_{k=0}^{\infty} \frac{\lambda^{k} \Gamma(k+t) \lambda^{k+r} \Gamma(k+r+t)}{(1+\lambda)^{k+t} \Gamma(t) k !(1+\lambda)^{k+r+t}(k+r) ! \Gamma(t)} \\
& =\frac{1}{(1+\lambda)^{2 t} \Gamma^{2}(t)} \sum_{k=0}^{\infty} \frac{\lambda^{2 k+r}}{(1+\lambda)^{2 k+r} k !(k+r) !} \int_{0}^{\infty} \mathrm{d} w \int_{0}^{\infty} \mathrm{d} z \mathrm{e}^{-w-z} w^{k+t-1} z^{k+r+t-1} \\
& =\frac{1}{(1+\lambda)^{2 t} \Gamma^{2}(t)} \int_{0}^{\infty} \int_{0}^{\infty} \mathrm{d} w \mathrm{~d} z \mathrm{e}^{-w-z} w^{t-(r / 2)-1} z^{(r / 2)+t-1} \sum_{k=0}^{\infty} \frac{((\lambda / 1+\lambda) \sqrt{w z})^{2 k+r}}{k !(k+r) !} \\
& =\frac{1}{(1+\lambda)^{2 t} \Gamma^{2}(t)} \int_{0}^{\infty} \int_{0}^{\infty} \mathrm{e}^{-w-z} w^{t-(r / 2)-1} z^{(r / 2)+t-1} I_{r}\left(\frac{2 \lambda \sqrt{w z}}{1+\lambda}\right) \\
& =\int_{0}^{\infty} \int_{0}^{\infty} \mathbb{P}\left\{N_{1}^{u}(1)-N_{2}^{y}(1)=r\right\} \mathrm{e}^{-(u+y / \lambda)} \frac{(u y)^{t-1} \mathrm{~d} u \mathrm{~d} y}{\lambda^{2 t} \Gamma^{2}(t)} \\
& =\mathbb{E} \mathbb{P}\left\{N_{1}^{U}(1)-N_{2}^{Y}(1)=r\right\} \text {, }
\end{aligned}
$$


where $U$ and $Y$ are independent gamma RVs with parameters 1 and $t$, and $I_{0}(x)$ is a Bessel function. For the reader's convenience, we recall that the Skellam distribution reads

$$
\mathbb{P}\left\{N_{1}^{\lambda}(t)-N_{2}^{\beta}(t)=r\right\}=\mathrm{e}^{-(\beta+\lambda) t}(\lambda / \beta)^{(r / 2)} I_{|r|}(2 t \sqrt{\lambda \beta}), \quad r \in \mathbb{Z},
$$

for independent Poisson processes $N_{1}^{\lambda}, N_{2}^{\beta}$, with rate $\lambda$ and $\beta$, respectively.

\section{Acknowledgement}

The authors are grateful to the anonymous referee for all suggestions which substantially improved the presentation of the paper.

\section{References}

BEgHin, L. (2015). Fractional gamma and gamma-subordinated processes. Stoch. Anal. Appl. 33, 903-926.

Brix, A. (1999). Generalized gamma measures and shot-noise Cox processes. Adv. Appl. Prob. 31, 929-953.

Cahoy, D. O. and Polito, F. (2012). On a fractional binomial process. J. Statist. Phys. 146, 646-662.

Di Crescenzo, A., Martinucci, B. and ZaCKs, S. (2015). Compound Poisson process with a Poisson subordinator. J. Appl. Prob. 52, 360-374.

Kozubowski, T. J. AND PodGórski, K. (2009). Distributional properties of the negative binomial Lévy process. Prob. Math. Statist. 29, 43-71.

Kreer, M., Kizilersü, A. ANd Thomas, A. W. (2014). Fractional Poisson processes and their representation by infinite systems of ordinary differential equations. Statist. Prob. Lett. 84, 27-32.

Kumar, A., Nane, E. and Vellaisamy, P. (2011). Time-changed Poisson processes. Statist. Prob. Lett. 81, $1899-1910$.

LASKIN, N. (2003). Fractional Poisson process. Chaotic transport and complexity in classical and quantum dynamics. Commun. Nonlinear Sci. Numer. Simul. 8, 201-213.

LiEB, E. H. (1990). The stability of matter: from atoms to stars. Bull. Amer. Math. Soc. 22, 1-49.

Meerschaert, M. M., Nane, E. and Vellaisamy, P. (2011). The fractional Poisson process and the inverse stable subordinator. Electron. J. Prob. 16, 1600-1620.

Orsingher, E. AND Polito, F. (2012a). Compositions, random sums and continued random fractions of Poisson and fractional Poisson processes. J. Statist. Phys. 148, 233-249.

Orsingher, E. And Polito, F. (2012b). The space-fractional Poisson process. Statist. Prob. Lett. 82, 852-858.

SChilling, R. L., Song, R. AND VondRAČEK, Z. (2010). Bernstein Functions: Theory and Applications. De Gruyter, Berlin.

Vellaisamy, P. And Maheshwari, A. (2014). Fractional negative binomial and Polya processes. Preprint. Available at http://arXiv.org/abs/1306.2493. 